\title{
8K ultra-high-definition microscopic camera for ophthalmic surgery
}

This article was published in the following Dove Press journal:

Clinical Ophthalmology

\author{
Hiromasa Yamashita ${ }^{1,2}$ \\ Kenkichi Tanioka ${ }^{2}$ \\ Goichiro Miyake ${ }^{3}$ \\ Ichiro Ota ${ }^{3}$ \\ Toru Noda ${ }^{4}$ \\ Kensaku Miyake ${ }^{3}$ \\ Toshio Chiba ${ }^{1,2}$ \\ 'Kairos Co. Ltd., Tokyo, Japan; ${ }^{2}$ Medical \\ Imaging Consortium, Tokyo, Japan; \\ ${ }^{3}$ Miyake Eye Hospital, Nagoya, Japan; \\ ${ }^{4}$ Tokyo Medical Center, Tokyo, Japan
}

Correspondence: Hiromasa Yamashita Kairos Co., Ltd., Sumitomo Fudosan Shiba Building 4, 4F, 2-13-4, Shiba, Minato-ku, Tokyo, 105-0014, Japan

Tel +8 I 354399583

Fax+8I 334532397

Email yamashita@kairos-8k.co.jp
Background: We have developed a new compact lightweight $8 \mathrm{~K}$ ultra-high-definition (UHD; $7,680 \times 4,320$ pixels) camera and started medical application with an ophthalmic surgical microscope which is interchangeable with the conventional high-definition $(1,920 \times 1,080$ pixels $) / 4 \mathrm{~K}$ UHD $(3,840 \times 2,160$ pixels $)$ microscopic camera.

Methods: We did a feasibility study to apply our 8K UHD microscope in cataract surgery, glaucoma surgery and vitreous surgery using pig cadaver eyes. The 8K UHD microscope comprises a surgical microscope, a camera adaptor with relay lenses, an 8K UHD camera and an $8 \mathrm{~K}$ UHD LCD to share the 8K UHD images with all surgical staff in real time.

Results: In ophthalmic surgeries, higher resolution images than conventional microscopic cameras were obtained with $8 \mathrm{~K}$ UHD LCD equivalent to the observation through the microscopic eye pieces.

Conclusion: Based on the results of this feasibility study, clinical trials on human ophthalmic surgery using the new $8 \mathrm{~K}$ UHD microscopic camera should be conducted in the near future.

Keywords: $8 \mathrm{~K}$ ultra-high-definition camera, surgical microscope, cataract surgery, glaucoma surgery, vitreoretinal surgery, heads-up surgery

\section{Introduction}

Japan Broadcasting Corporation (NHK) developed 8K ultra-high-definition (UHD) broadcasting technologies and achieved a series of devices specific for 8K UHD imaging in 2002. 8K UHD broadcasting system offers panoramic images of a resolution 16-fold higher than the images obtained with the high-definition (HD) technology. ${ }^{1-4}$ There are heightening expectations in recent years for important contributions of $8 \mathrm{~K}$ UHD technology to innovative medical imaging in advanced image-guided diagnosis and treatment.

In ocular microsurgery, high-performance microscopes have been strongly demanded for the highest precision observation and operation. Ophthalmology microscopes attached with HD/4K UHD camera have been already commercialized. However, the image quality of the HD/4K UHD camera is inferior to that of the surgeons' eyes' view through the microscopic optical system. The first experiment in the world to apply 8K UHD in microscopic surgeries of cataract, glaucoma, and vitreoretinal was successfully conducted by the Medical Imaging Consortium in Miyake Eye Hospital (Nagoya, Japan) in December 2014 using our prototype 8K UHD camera, which was originally developed for an 8K UHD endoscope. ${ }^{5}$ Subsequently, we have developed a new compact lightweight 8K UHD camera (Kairos Co., Ltd., KairoScope-E, Tokyo, Japan) in $2017^{6}$ and started medical application of ophthalmic surgical microscope which is interchangeable with the conventional $\mathrm{HD} / 4 \mathrm{~K}$ camera. 
In this paper, we report the results of the experiment to apply the new $8 \mathrm{~K}$ UHD microscopic camera in ophthalmic surgeries using pig cadaver eyes.

\section{Materials and methods 8K UHD microscopic camera for ophthalmic surgery}

Figure 1 shows our new compact lightweight 8K UHD microscopic camera for ophthalmic surgery. The $8 \mathrm{~K}$ UHD camera is interchangeable with the conventional $\mathrm{HD} / 4 \mathrm{~K}$ camera mounted on the ophthalmology microscopes. The dimensions of the $8 \mathrm{~K}$ UHD camera are $75 \mathrm{~mm}(\mathrm{~W}) \times 75 \mathrm{~mm}(\mathrm{H}) \times 170$ $\mathrm{mm}(\mathrm{L})$ and the weight is $370 \mathrm{~g}$. The $8 \mathrm{~K}$ UHD camera has a super-35-mm complementary metal oxide semiconductor imaging sensor and its specifications are shown in Table 1. Especially, sensitivity of the imaging sensor is about four times superior to that of our prototype $8 \mathrm{~K}$ UHD camera.

The prototype $8 \mathrm{~K}$ UHD camera was too large to be mounted on a surgical microscope. On the contrary, our new 8K UHD camera size is nearly one fifth of the prototype one and can be attached to the surgical microscope without any mechanical interference on the microscope. Furthermore, as our compact $8 \mathrm{~K}$ UHD camera is light, it can be attached to both table top and stand type microscopes without losing weight balance.

The optical system of an 8K UHD microscope comprises a surgical microscope, a camera adaptor with relay lenses, and an $8 \mathrm{~K}$ UHD camera. Half quantity of the light from one ray path for the right eye is guided to the $8 \mathrm{~K}$ UHD camera through the adaptor optics. The adaptor optics images the

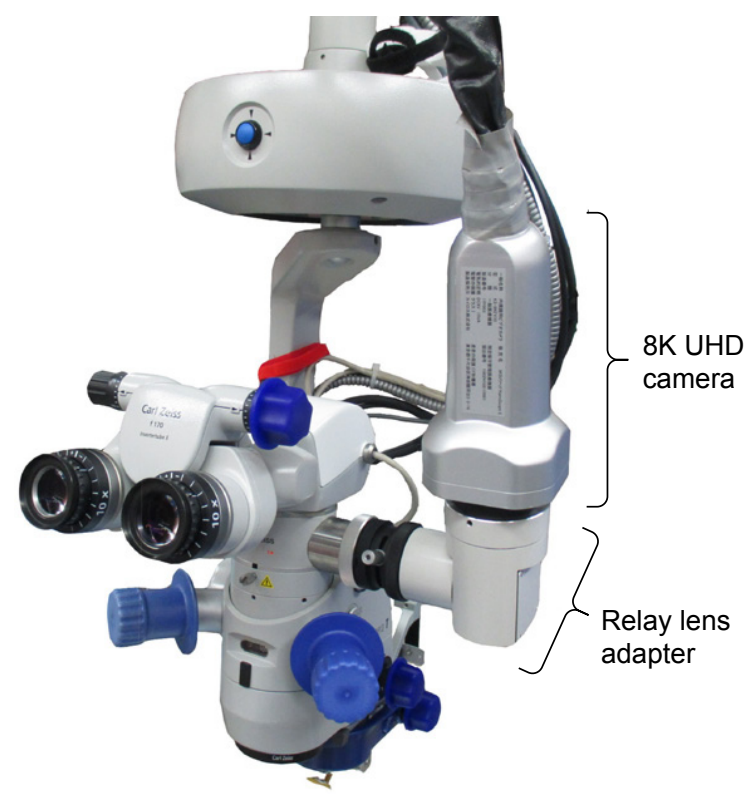

Figure I Our new 8K UHD camera mounted on a surgical microscope with a relay lens adapter.

Abbreviation: UHD, ultra-high-definition.
Table I 8K UHD CMOS imaging sensor specifications

\begin{tabular}{ll}
\hline Parameter & Specifications \\
\hline Optical format & Super $35 \mathrm{~mm}$ \\
Pixel area & $24.6 \mathrm{~mm}(\mathrm{H}) \times 13.9 \mathrm{~mm}(\mathrm{~V})$ \\
Pixel unit size & $3.2 \mu \mathrm{m} \times 3.2 \mu \mathrm{m}$ \\
Number of active pixels & $7,680(\mathrm{H}) \times 4,320(\mathrm{~V})$ \\
Sensitivity & $>1.6 \mathrm{~V} / \mathrm{lux}$-second \\
Dynamic range & $>68 \mathrm{~dB}$ \\
Power consumption & $<3 \mathrm{~W}$ \\
Chroma & Mono and Bayer RGB \\
\hline
\end{tabular}

Abbreviations: CMOS, complementary metal oxide semiconductor; RGB, red, green and blue; UHD, ultra-high-definition.

magnified surgical sites on the pixels of the $8 \mathrm{~K}$ UHD camera with an optimal focal length of $289 \mathrm{~mm}$.

\section{Feasibility study using pig cadaver eyes}

We tried cataract surgery (continuous circular capsulorhexis [CCC], phacoemulsification, and intraocular lens insertion), glaucoma surgery (observation through a gonioscope and sclera flap creating), and vitreous surgery (guillotine cut and peeling) using pig cadaver eyes in Miyake Eye Hospital. Our new 8K UHD camera was attached to an assistant microscope installation part of a commercialized ophthalmology microscope (Carl Zeiss, Zeiss Lumera T, Oberkochen, Germany) suspended from the ceiling. The intensity of microscopic illumination was between 40,000 and 47,000 lux in cataract and glaucoma surgery. In vitreous surgery, the intensity of endoillumination and chandelier light was about 33,000 and 50,000 lux, respectively. A 70-inch 8K UHD monitor (LV-70002; Sharp Corporation, Osaka, Japan) was placed in front of a surgeon. Figure 2 shows the operating room site using an $8 \mathrm{~K}$ UHD microscopic camera for ophthalmic surgeries.

\section{Results}

Figure 3 shows examples of the anterior eye segment - a comparison between the surgical field images of pig cadaver eyes in cataract surgery (phacoemulsification) obtained with the prototype $8 \mathrm{~K}$ UHD microscopic camera (Figure $3 \mathrm{~A}$ ) and that by the new 8K UHD microscopic camera (Figure 3B). In Figure 3A, since the brightness of the image was insufficient with the open aperture, a manual gain-up was added up to $+15 \mathrm{~dB}$ by digital processing. The bright image of Figure $3 \mathrm{~B}$ was obtained without an automatic digital gain-up and there was a margin of $1 / 2$ to $1 / 3$ of the aperture scale. Therefore, effectively ten times more sensitive image was obtained, so that the automatic gain control is available, which allows constant brightness even when changing light and dark areas. In addition, even aspirated groove texture of crystalline lens was highly clear with a fine difference like a shading change in Figure 3B. 


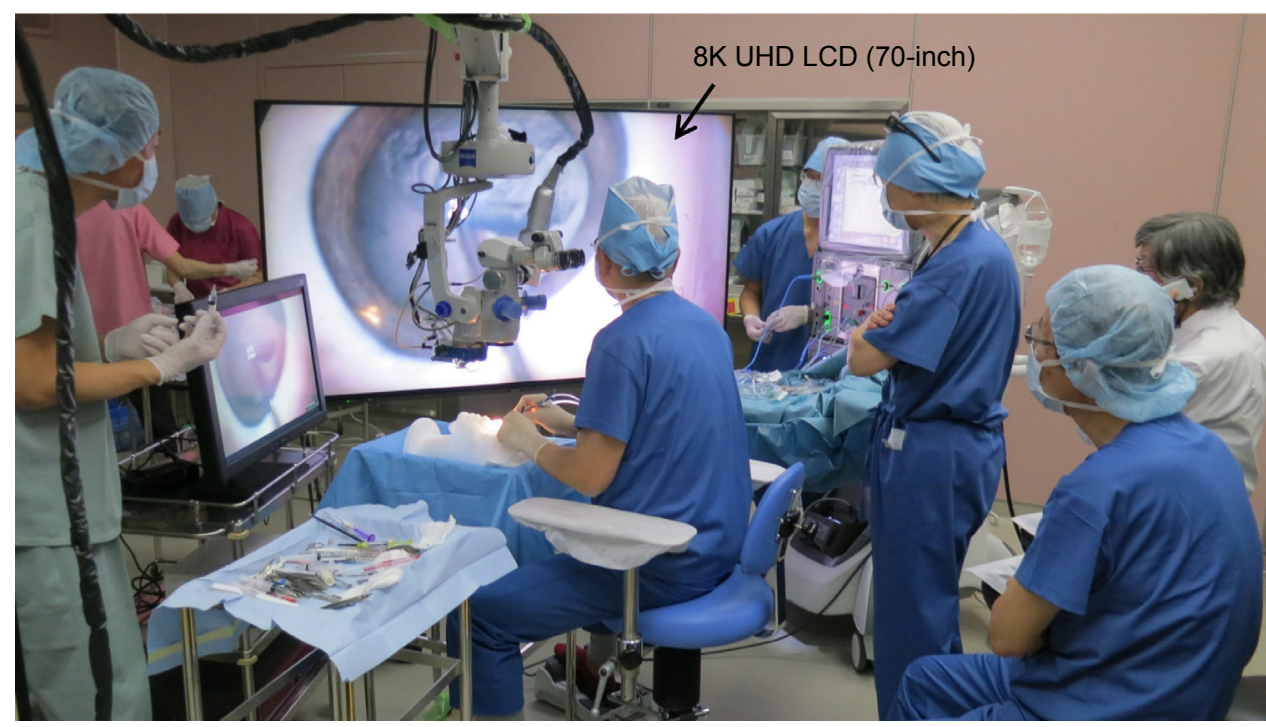

Figure $28 \mathrm{~K}$ UHD microscopic camera for ophthalmic surgery in the operating room site. Abbreviation: UHD, ultra-high-definition.

Figure 4 shows a comparison between the surgical field images of pig cadaver eyes in glaucoma surgery (observation through a gonioscope and creating scleral flap) obtained with the prototype $8 \mathrm{~K}$ UHD microscopic camera (Figure 4A) and that with the new 8K UHD microscopic camera (Figure 4B). Similar to the 8K UHD images in cataract surgery (Figure 3B), the images obtained with the new $8 \mathrm{~K}$ UHD microscopic camera in glaucoma surgery (Figure 4B) were brighter than those obtained with the prototype $8 \mathrm{~K}$ UHD microscopic camera (Figure 4A). In Figure 4B, the fine microstructures of trabecular meshwork and ciliary body can be clearly observed with brighter $8 \mathrm{~K}$ UHD resolution, and the quality was sufficiently high and equivalent to the microscopic direct observation through eyepieces.

Figure 5 shows examples of posterior eye segment $-\mathrm{a}$ comparison between surgical field images of pig cadaver eyes in vitreoretinal surgery obtained with the prototype $8 \mathrm{~K}$ UHD microscopic camera (Figure 5A) and that with the new 8K UHD microscopic camera (Figure 5B). Posterior eye segment was illuminated by a chandelier endoillumination fiber, and quantity of illumination light for posterior eye segment was quite limited. High sensitivity of the new 8K UHD microscopic camera caused illumination of larger range (Figure 5B) than the prototype 8K UHD microscopic camera (Figure 5A).

Supplementary Video S1 shows detail of Figures 3-5 with HD resolution down-converted from an original $8 \mathrm{~K}$ UHD video.

\section{Discussion}

We have developed a new compact and lightweight $8 \mathrm{~K}$ UHD microscopic camera for ophthalmic surgery, which is interchangeable with the conventional $\mathrm{HD} / 4 \mathrm{~K}$ microscopic
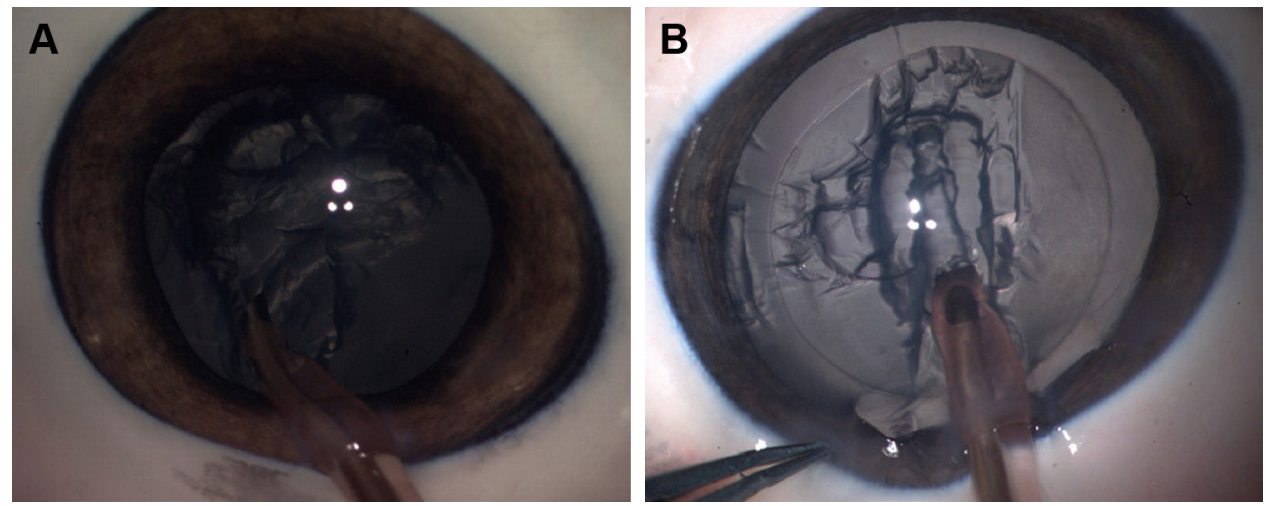

Figure 3 The comparison of surgical field images of pig cadaver eye in cataract surgery (phacoemulsification). (A) Image obtained with the prototype 8K microscopic camera and (B) image obtained with the new 8K UHD microscopic camera.

Abbreviation: UHD, ultra-high-definition. 

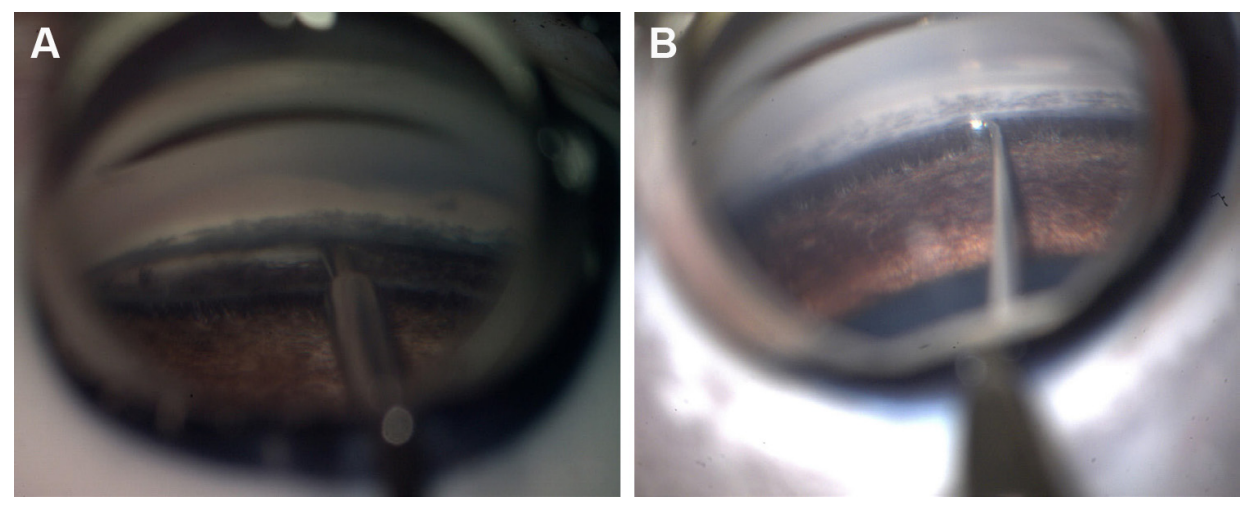

Figure 4 The comparison of surgical field images of pig cadaver eye in glaucoma surgery. (A) Images obtained with the prototype 8K microscopic camera and (B) images obtained with the new 8K UHD microscopic camera through a gonioscope.

Abbreviation: UHD, ultra-high-definition.

camera. We did the feasibility study to apply our microscopic camera in ophthalmic surgeries using pig cadaver eyes, and images of sufficiently high quality could be obtained with 8K UHD LCD equivalent to the microscopic observation through the eye pieces for not only a surgeon but also other surgical staffs in real time.

Surgeons and other surgical staffs reported the following positive evaluations of the new $8 \mathrm{~K}$ UHD microscopic camera for ophthalmic surgery:

- The new compact and lightweight 8K UHD camera with higher sensitivity deserves a sufficient evaluation from the viewpoint of surgical suitability.

- The new 8K UHD camera can increase the quantity and quality of microscopic digital images on the monitor, and the $8 \mathrm{~K}$ UHD images are equivalent to real microscopic observation through eyepieces.

- Brightness of the image approached practical use for $8 \mathrm{~K}$ UHD microscopic camera.

- In glaucoma surgery, creating a sclera flap might not require stereoscopic vision so much because an $8 \mathrm{~K}$ UHD image is easier to be seen than an image through a micro- scope with natural three-dimensional effect in a wide range, which might be possible for near-future "heads-up surgery" without observation through microscopic optical system (Figure 6).

- In cataract CCC, texture, thickness, and wrinkles of the capsule can be recognized easily.

- In phacoemulsification, switching from normal illumination method to transillumination method causes easier recognition of the aspirated groove depth of crystalline lens.

- In vitreous surgery, information about fine blood vessels and flow, membrane, and bleeding fiber tissue in the fundus might be obtained.

On the other hand, surgeons also noted the following challenges because of insufficient sensitivity of the $8 \mathrm{~K}$ UHD image sensor:

- Because enough light did not reach from the light source of the microscope, it was difficult to observe the posterior portion (vitreous body) of the eye, followed by the anterior portion (cataract) and surface portion (sclera), due to the structure of the eye ball with several optical systems that create aberrations.
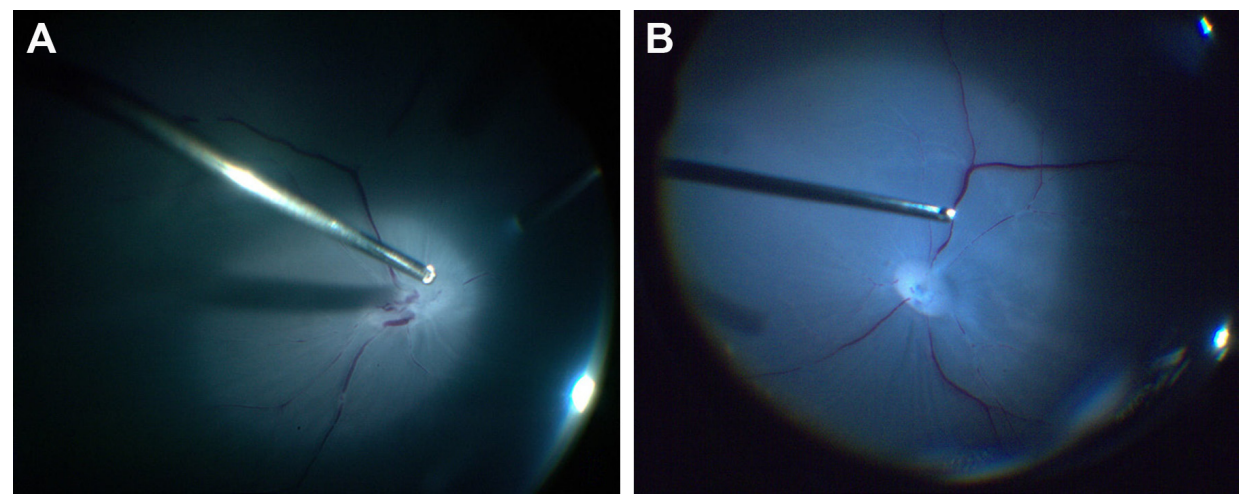

Figure $\mathbf{5}$ Comparison of surgical field images of pig cadaver eye in vitreoretinal surgery. (A) Images obtained with the prototype 8K microscopic camera and (B) images obtained with the new 8K UHD microscopic camera. Retinal capillary vessels were illuminated by a chandelier endo-illumination fiber.

Abbreviation: UHD, ultra-high-definition. 




Figure 6 8K UHD image in creating a sclera flap of pig cadaver eye. Abbreviation: UHD, ultra-high-definition.

- Improvement of sensitivity of the new 8K UHD camera made it easier to focus on the operative field with suitable adjustment of the diaphragm for the adaptor optics lens and deeper depth of field; however, focusing is still more difficult than observing through the microscopic eye pieces.

\section{Conclusion}

The simulated surgeries using pig cadaver eye and comparison with the prototype $8 \mathrm{~K}$ UHD microscopic camera have demonstrated the practicality of the new compact and lightweight $8 \mathrm{~K}$ UHD microscopic camera for ophthalmic surgery in clinical practice and confirmed new possible use of the 8K UHD microscopic camera images. Based on the results of feasibility study (glaucoma surgery, cataract surgery, and vitreous surgery), clinical trials on human ophthalmic surgery using the new $8 \mathrm{~K}$ UHD microscopic camera might be conducted in the near future.

\section{Acknowledgment}

A part of this work was supported by the Japan Agency for Medical Research and Development (AMED) grant "Developing smart treatment centers with balanced safety and enhanced medical efficiency (8K UHD endoscope systems)".

\section{Author contributions}

All authors contributed toward data analysis, drafting and critically revising the paper, gave final approval of the version to be published, and agree to be accountable for all aspects of the work. Hiromasa Yamashita was in charge of optimal combination of the $8 \mathrm{~K}$ UHD camera and a surgical microscope with a relay lens adapter and collection and assembly of the 8K UHD microscopic images. Kenkichi Tanioka was in charge of collection and assembly of the 8K UHD microscopic images and adjustment of aperture of the relay lens adapter during surgical operations. Goichiro Miyake was in charge of surgical operations during cataract surgery, glaucoma surgery, and vitreous surgery using pig cadaver eyes in Miyake Eye Hospital. Ichiro Ota was in charge of the planning, setting, and evaluation of this feasibility study. Toru Noda was in charge of an optimal design of the relay lens adapter to connect between microscopic optical system and the 8K UHD camera. Kensaku Miyake was in charge of final approval of the feasibility study in Miyake Eye Hospital. Toshio Chiba was the principal investigator of this feasibility study and was in charge of final approval of the article.

\section{Disclosure}

Hiromasa Yamashita is the Chief R\&D Executive of Kairos Co., Ltd. The authors report no other conflicts of interest in this work.

\section{References}

1. Shimamoto H, Yasue T, Kitamura K, et al. A Compact 120 Frames/ sec UHDTV2 Camera with $35 \mathrm{~mm}$ PL Mount Lens. SMPTE Motion Imaging J. 2014;123(4):21-28.

2. Sugawara M, Kanazawa M, Mitani K, Shimamoto H, Yamashita T, Okano F. Ultrahigh-Definition Video System with 4000 Scanning Lines. SMPTE Motion Imaging J. 2003;112(10-11):339-346.

3. Sugawara M, Emoto M, Masaoka K, Nishida Y, Shishikui Y. Super hi-vision for the next generation television. ITE Trans MTA. 2013; 1(1):27-33.

4. Yamashita T, Masaoka K, Ohmura K, Emoto M, Nishida Y, Sugawara M. Super Hi-Vision Video Parameters for Next-Generation Television. SMPTE Motion Imaging J. 2012;121(4):63-68.

5. Yamashita H, Aoki H, Tanioka K, Mori T, Chiba T. Ultra-high definition (8K UHD) endoscope: our first clinical success. Springerplus. 2016; 5(1):1445.

6. Yamashita H, Tanioka K, Chiba T. A historical game-changer: The world's smallest 8K UHD endoscope: current state of the art. Proc SPIE. 2018; 10557:105570A. 


\section{Supplementary material}

Video SI Detail of Figures 3-5 with HD resolution down-converted from an original 8K UHD video.

Abbreviations: HD, high-definition; UHD, ultra-high-definition.

\section{Publish your work in this journal}

Clinical Ophthalmology is an international, peer-reviewed journal covering all subspecialties within ophthalmology. Key topics include: Optometry; Visual science; Pharmacology and drug therapy in eye diseases; Basic Sciences; Primary and Secondary eye care; Patient Safety and Quality of Care Improvements. This journal is indexed on

PubMed Central and CAS, and is the official journal of The Society of Clinical Ophthalmology (SCO). The manuscript management system is completely online and includes a very quick and fair peer-review system, which is all easy to use. Visit http://www.dovepress.com/ testimonials.php to read real quotes from published authors. 